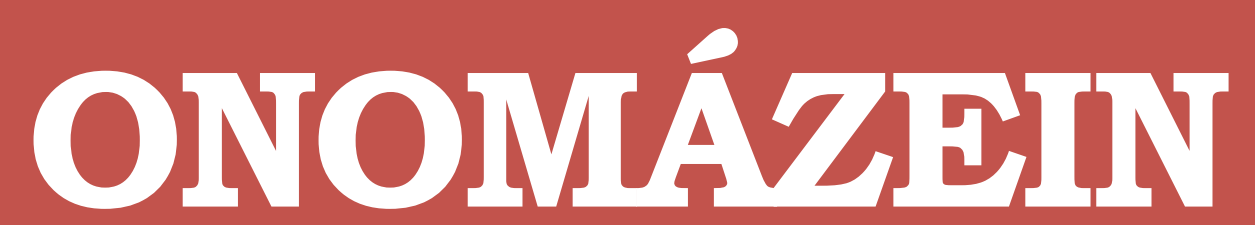

Revista de lingüística, filología y traducción

\title{
Propuesta para el análisis de la evolución literaria de un autor mediante el uso de herramientas propias de la lingüística de corpus: el ejemplo de Pérez Galdós
}

Analyzing the Literary Evolution of an Author Using Corpus-Linguistic Tools: the Case of Pérez Galdós

\section{Guadalupe Nieto Caballero \\ Universidad de Extremadura \\ España}




\section{Resumen}

En el presente artículo se realiza una propuesta para analizar la evolución estilística de un autor a lo largo de su trayectoria, usando herramientas propias de la lingüística de corpus. Para ello se ha utilizado la obra narrativa de Pérez Galdós (ca. 6,4 millones de palabras), que ha sido procesada con el software de concordancias WordSmith Tools 7 (Scott, 2016). El análisis se centra en la proyección del discurso por parte de los narradores de las distintas novelas. En concreto, se ha analizado la forma verbal dijo, que ha revelado la frecuencia de uso de distintas estrategias de representación del discurso en distintos estadios de la trayectoria de Galdós, lo que ha permitido distinguir dos fases bien diferenciadas en su estilo. Posteriormente, se han analizado algunas de las opciones ejercidas sistemáticamente por el autor en el uso dijo, que definen claramente su estilo y contribuyen a trazar una evolución en su trayectoria. El artículo pretende ilustrar el potencial de los estudios de estilística de corpus en el ámbito de la literatura en español, un área todavía escasa de análisis que adopten este tipo de enfoque.

Palabras clave: estilo literario; evolución; Benito Pérez Galdós; estilística de corpus.

\section{Abstract}

This article looks into the potential of corpus linguistic approaches in the analysis of the evolution of a literary author's style throughout his career. To do so, the fictional narratives of Pérez Galdós (c. 6.4 million words) have been scrutinized. They have been processed with the software tool WordSmith Tools (Scott, 2016). The analysis has focused on the reporting of characters' verbal discourse by the narrators of Galdós's novels, paying special attention to the speech verb dijo. The corpus-based approach used to carry out the analysis has made it possible to quantify the frequency with which Galdós uses different discourse presentation strategies at different stages of his career, which has revealed a clear evolution as far as his craftsmanship is concerned. Due to length constraints, we have focused on his early novels, analyzing the role of dijo as a core element in textual building blocks relevant to the creation of fictional universes and fictional personalities. The analysis is meant to make a contribution to the still-emerging field of corpus stylistics in Spanish, illustrating how the analysis of literary works can benefit greatly from the use of innovative corpus tools.

Keywords: literary style; evolution; Benito Pérez Galdós; corpus stylistics. 


\section{Introducción}

En el presente artículo se realiza una propuesta para analizar la evolución estilística de un autor a lo largo de su trayectoria, utilizando herramientas propias de la lingüística de corpus. Para ello se ha utilizado la obra narrativa de Pérez Galdós (ca. 6,4 millones de palabras), que ha sido procesada con el software de concordancias WordSmith Tools 7 (Scott, 2016). El análisis se puede englobar dentro de la estilística de corpus (Mahlberg, 2016), una disciplina en la que se combinan enfoques propios de la crítica literaria tradicional y la mencionada lingüística de corpus. Aunque las metodologías de corpus no resultan novedosas en los estudios literarios en general' ${ }^{1}$, sí que lo son en el análisis de textos literarios en español². En este sentido, el artículo pretende contribuir a demostrar el potencial de este tipo de enfoque en los estudios literarios de obras en lengua española, y abrir así nuevas vías de análisis que hasta ahora no han sido exploradas en la exégesis de autores en este idioma. Desde un punto de vista exclusivamente literario, por otra parte, el propósito del estudio reside en ser capaces de analizar aspectos del estilo de Galdós que, debido a la falta de herramientas adecuadas, no han podido ser identificados con anterioridad —o al menos no han podido ser analizados de forma metódica- De manera más concreta, el artículo se centra en la proyección del discurso de los personajes a lo largo de sus más de setenta novelas por parte de los diferentes narradores de estas. El análisis gira, por cuestiones tanto de espacio como de concreción metodológica, en torno a la forma verbal dijo, verbo de habla por excelencia en la proyección del discurso en el género novelesco. Como se podrá comprobar, el análisis sistemático del uso que Galdós hace de esta forma verbal revela aspectos estilísticos relevantes en la obra del autor canario. Gracias al procesamiento de sus novelas con un software de concordancias, además, el grado de prominencia de tales aspectos puede ser calibrado a lo largo de su trayectoria, lo que permitirá distinguir dos fases bien diferenciadas en el estilo del autor y trazar una evolución.

El artículo se organiza como sigue. En primer lugar, se realiza una breve contextualización del marco de estudio, en la que se apunta tanto la razón para escoger la figura de Galdós

1 Estas metodologías, de hecho, vienen empleándose con frecuencia desde finales del siglo pasado (cf. Lancashire, 1993). La evolución en la literatura a través del estudio de patrones estilísticos propios de cada época también ha sido objeto de análisis con metodologías de corpus (cf. Hughes, Foti, Krakauer y Rockmore, 2012), así como el análisis de la evolución literaria de un autor concreto que aquí nos ocupa. La figura de Dickens y su desarrollo como novelista, por ejemplo, ha sido explorada utilizando enfoques de estilística de corpus (cf. Mahlberg y Smith, 2012; Mahlberg, Smith y Preston, 2013).

2 Aunque escasas, existen notables excepciones en este sentido. Sirva como ejemplo la investigación llevada a cabo por Martínez-Gamboa en torno a patrones cuantitativos en novelas chilenas desde el siglo XIX al siglo XXI (Martínez-Gamboa, 2015) o su análisis del Big Data en el campo de las humanidades digitales (Martínez-Gamboa, 2016). 
como la evolución que habitualmente se traza en su trayectoria en términos de estrategias de representación del discurso (apartado 2). En el apartado 3 se presenta el corpus de estudio galdosiano y se abordan cuestiones relacionadas con su construcción. El apartado 4, que comprende la parte analítica, se divide en tres subapartados. Tras una introducción al tema objeto de estudio y la comparación de la forma verbal dijo en la obra de Galdós con respecto a la novela realista española en general, se realiza un análisis de la distribución de esta forma en la producción del autor canario, lo que nos permitirá distinguir dos fases bien diferenciadas en su trayectoria (apartado 4.1). Debido a su prominencia en la fase inicial, el análisis del valor estilístico de esta forma se centra fundamentalmente en las novelas de la primera etapa. En concreto, se aborda cómo la forma verbal dijo se asocia con otros elementos y forma bloques textuales de los que Galdós se sirve para construir sus universos ficticios (apartado 4.2), así como las funciones que estos bloques textuales desempeñan en novelas concretas como recurso de caracterización (apartado 4.3). El artículo finaliza con una breve reflexión en torno al potencial de los estudios de estilística de corpus en el ámbito de la literatura en español, un área todavía escasa de análisis que adopten este tipo de enfoque.

\section{Marco de estudio}

La razón para escoger a Galdós como objeto de análisis del presente estudio tiene que ver fundamentalmente con que se trata de uno de los novelistas españoles más laureados de siempre - quizá solo por detrás de Cervantes-. Galdós fue, además, un escritor muy prolijo, con más de setenta novelas en su haber, lo que lo convierte en un autor ideal para ser analizado con herramientas de corpus, pues cabe esperar que estas revelen patrones tanto formales como funcionales que hasta ahora han pasado desapercibidos para la crítica y que ayuden a una mejor comprensión de su estilo. Además, pertenece a una de las épocas doradas de la novela española: el Realismo. Este hecho hace posible que su obra pueda compararse con la de sus contemporáneos, lo que permitirá calibrar la representatividad de los rasgos que se analizan en relación a la pléyade de autores con los que compartió plana.

La razón para centrarnos en la proyección del discurso es que, con el paso del tiempo, Galdós evolucionó hacia un estilo cada vez más psicológico, en donde el plano mental de sus personajes cobra mayor importancia. Desde un punto de vista estilístico, esto se materializa en el uso de nuevas técnicas narrativas para dar voz a sus personajes, como el monólogo interior o el estilo indirecto libre (cf. Gullón, 1960; Pedraza Jiménez y Rodríguez Cáceres, 1983: 795; Andreu, 1989). Con estas estrategias de representación del discurso no solo se minimiza la figura del narrador — de importancia cardinal en sus primeras obras, como se comenta más adelante-, sino que se "consigue una vía de ingreso hacia la interioridad del personaje" (Vargas Llosa, 1975: 239), uno de los pilares del Naturalismo, el movimiento que reemplazaría al Realismo y que para muchos Galdós fue abrazando a medida que se sucedieron sus novelas (Benítez, 
1992: 260). Normalmente, los autores a los que se suele citar como ejemplo de virtuosismo en el uso del estilo indirecto libre son de primera mitad del XX, como Henry James, Alain Proust o James Joyce. En la literatura española también encontramos a novelistas como Luis MartínSantos o Miguel Delibes. Antes de ellos, no obstante, ya estuvo la figura de Galdós, a quien se puede considerar un precursor de lo que más tarde se convertiría en tendencia. Sin embargo, el estilo de Galdós, dominado por el monólogo interior y el estilo indirecto libre, como se intenta ilustrar en este análisis mediante el uso de herramientas de corpus, no es idiosincrásico de toda su obra, sino el resultado de una evolución literaria a medida que se suceden sus trabajos. En el ejemplo (1) se muestra un fragmento perteneciente a La desheredada, publicada en 1881. Como se comenta en el apartado 4, esta novela marcará un punto de inflexión en el estilo del autor. De hecho, es considerada por parte de la crítica como una de las primeras novelas naturalistas españolas (cf. Sotelo, 2013: 52). El fragmento corresponde al inicio de la novela. Precisamente por la técnica del monólogo interior que en él se advierte -y que está presente en varios puntos de la obra-, Clarín se referiría a esta novela como un ejemplo del "subterráneo hablar de la conciencia" (en Gutiérrez Carbajo, 2013: 263). Además de monólogos interiores y estilo indirecto libre, en La desheredada Galdós también utiliza diferentes técnicas narrativas innovadoras, como el multiperspectivismo, la segunda persona narrativa o la inclusión de capítulos enteros dialogados. Estos recursos forman parte de un nuevo estilo, articulado en gran medida en torno al estudio psicológico de los personajes de las novelas.

(1) ... ¿Se han reunido todos los ministros?... ¿Puede empezar el Consejo?... iEl coche, el coche, o no llegaré a tiempo al Senado!... Esta vida es intolerable... ¡Y el país, ese bendito monstruo con cabeza de barbarie y cola de ingratitud, no sabe apreciar nuestra abnegación, paga nuestros sacrificios con injurias, y se regocija de vernos humillados! Pero ya te arreglaré yo, país de las monas. ¿Cómo te Ilamas? Te Ilamas Envidiópolis, la ciudad sin alturas; y como eres puro suelo, simpatizas con todo lo que cae... ¿Cuánto va? Diez millones, veinticuatro millones, ciento sesenta y siete millones, doscientas treinta y tres mil cuatrocientas doce pesetas con setenta y cinco céntimos...; esa es la cantidad. Ya no te me olvidarás, pícara; ya te pillé, ya no te me escapas, ioh cantidad temblorosa, escurridiza, inaprehensible, como una gota de mercurio! Aquí te tengo dentro del puño, y para que no vuelvas a marcharte, jugando, al caos del olvido, te pongo en esta gaveta de mi cerebro, donde dice: Subvención personal... Permítame Su Señoría que me admire de la despreocupación con que Su Señoría y los amigos de Su Señoría confiesan haber infringido la Constitución... No me importan los murmullos. Mandaré despejar las tribunas... iA votar, a votar! ¿Votos a mí? ¿Queréis saber con qué poderes gobierno? Ahí los tenéis: se cargan por la culata. He aquí mis votos: me los ha fabricado Krupp... Pero ¿qué ruido es este? ¿Quién corretea en mi cerebro? ¡Eh!, ¿quién anda arriba?... Ya, ya; es la gota de mercurio, que se ha salido de su gaveta... (La desheredada, capítulo 1)3.

3 Todos los ejemplos citados en el artículo han sido extraídos de una versión electrónica de los textos. Por lo tanto, en lugar de la paginación, únicamente se ofrece como referencia el capítulo en el que aparecen. 
Sin embargo, este Galdós, cada vez más presente en la fase tardía, poco tiene que ver en realidad con el de la época inicial desde un punto de vista estilístico. En sus comienzos, la representación del discurso verbal prima sobre la representación del discurso mental, y en lugar de estrategias caracterizadas por el uso de frases cortas, ideas espontáneas y discurso poco organizado, como ocurre con el monólogo interior o el estilo indirecto libre, Galdós emplea un "estilo directo y conciso" (Andreu, 1989: 61). Sirva como botón de muestra el ejemplo (2), extraído de su ópera prima La fontana de Oro (el énfasis es mío):

(2) - ¿Se atreve usted a negarlo? - dijo Paz, dando algunos pasos hacia ella con el resplandor de la ira en los ojos.

-Yo... no — dijo Clara, retrocediendo con espanto-. Sí... sí lo niego — después añadió, haciendo un esfuerzo por calmarse y calmar a su juez-: Óigame usted, señora: yo le contaré la verdad; le diré lo que ha sido. Yo soy inocente; yo no he permitido...

—iJesús, Jesús! Yo no sirvo para estas cosas — clamó Salomé volviendo el rostro—. No puedo, no puedo oír esto.

—¿¿ué usted no ha permitido...? ¿Todavía tiene atrevimiento para negarlo?

—Yo... yo no niego — contestó la huérfana muy consternada-. Pero yo, ¿qué culpa tengo de que ese hombre...?

_-¿También le quiere usted disculpar a él? Esto nos faltaba que ver. No puede haber perdón para tanta alevosía. iPagar de este modo el asilo que le hemos dado sin merecerlo! Pero bien dije yo que de usted no podíamos sacar cosa buena.

- Señoras —dijo Clara deshaciéndose en lágrimas-, yo les juro a ustedes por Dios y por todos los santos, que por mí no ha entrado ningún hombre; que yo no soy culpable de todo eso que ustedes dicen. Yo se lo juro por Dios y por la Virgen (La fontana de Oro, capítulo 35).

Este ejemplo ilustra a la perfección el Galdós de la época inicial a la hora de proyectar el discurso de sus personajes. Mediante el uso de estilo directo, el habla de sus figuras aparece intercalada con la voz del narrador, que contribuye frecuentemente a puntualizar aspectos relacionados con el plano psicológico de los personajes (contestó la huérfana muy consternada, por ejemplo). Naturalmente, existen abundantes ejemplos de estilo directo en la fase tardía de su producción, del mismo modo que en novelas de su primera época también encontramos ejemplos de monólogo interior y de estilo indirecto libre como el mostrado en (1). Sin embargo, es cierto que existe una dinámica clara a medida que se suceden las novelas. Y esta comporta una serie de implicaciones estilísticas más allá del ámbito psicológico y la condición del autor canario de precursor del estilo indirecto libre que en ocasiones se le atribuye. En concreto, el patrón estructural del estilo directo que se advierte en el diálogo del ejemplo (2) es empleado por Galdós, sobre todo en la época inicial, como un recurso narratológico para lograr efectos que van más allá de la representación del discurso, como son la construcción del universo ficticio o la caracterización de los personajes a través del protagonismo de la voz del narrador. Este es el Galdós del que nos ocuparemos en este trabajo, pues es quizá el que menos atención ha recibido desde un punto de vista puramente estilístico. En concreto, el objetivo es ser capaz de medir, a través de la frecuen- 
cia en el uso de la forma verbal dijo, la presencia de ejemplos como los que se advierten en (2) a lo largo de la producción de Galdós, lo que permitirá ofrecer un mapa del cambio en el estilo de Galdós en lo que a la representación del discurso se refiere y ayudará a ilustrar la evolución desde el plano realista al naturalista que la crítica suele comentar en el marco del estilo del autor.

\section{El corpus de estudio}

Los textos que forman el corpus de estudio se han recopilado del repositorio digital de la Biblioteca Virtual Miguel de Cervantes (s. f.). Este repositorio, que tiene como objetivo la difusión de la cultura hispánica en general, cuenta con alrededor de doscientos mil registros bibliográficos, de los cuales sesenta mil son libros. La calidad de la versión digitalizada de los textos, fundamental en los estudios de corpus como el que aquí se realiza, está fuera de toda duda, pues la Biblioteca cuenta con un consejo científico que avala el rigor de los materiales alojados en el repositorio ${ }^{4}$. En el caso de Galdós, toda su producción literaria se encuentra digitalizada. Para llevar a cabo este análisis, se ha seleccionado su obra narrativa, con una extensión de más de seis millones de palabras, como se muestra en la tabla 1. Su narrativa breve no ha sido incluida en el corpus, por no tratarse de novelas propiamente dichas. Tampoco ha sido incluida su producción teatral, por no pertenecer al género narrativo.

\section{TABLA 1}

Novelas incluidas en el corpus de estudio

\begin{tabular}{lccc} 
& NOVELA & PALABRAS \\
\hline Trafalgar & EPISOdIOS NACIONALES. PRIMERA SERIE & \\
\hline La Corte de Carlos IV & 1873 & 51.453 \\
\hline El 19 de Marzo yel 2 de Mayo & 1873 & 139.656 \\
\hline Bailén & 1873 & 136.403 \\
\hline Napoleón en Chamartín & 1873 & 63.819 \\
\hline Zaragoza & 1874 & 153.971 \\
\hline Gerona & 1874 & 133.010 \\
\hline Cádiz & 1874 & 58.453 \\
\hline
\end{tabular}

4 Cabe destacar que este consejo científico está presidido por el actual director de la Real Academia Española, Darío Villanueva Prieto. 


\begin{tabular}{|c|c|c|}
\hline Juan Martín El Empecinado & 1874 & 63.757 \\
\hline La Batalla de los Arapiles & 1875 & 90.064 \\
\hline \multicolumn{3}{|c|}{ EPISODIOS NACIONALES. SEGUNDA SERIE } \\
\hline El equipaje del Rey José & 1875 & 61.043 \\
\hline Memorias de un cortesano de 1815 & 1875 & 51.324 \\
\hline La Segunda Casaca & 1876 & 65.663 \\
\hline El Grande Oriente & 1876 & 64.034 \\
\hline 7 de Julio & 1876 & 51.624 \\
\hline Los Cien Mil Hijos de San Luis & 1877 & 56.331 \\
\hline El Terror de 1824 & 1877 & 67.105 \\
\hline Un voluntario realista & 1878 & 66.867 \\
\hline Los Apostólicos & 1879 & 77.113 \\
\hline Un faccioso más y algunos frailes menos & 1879 & 79.761 \\
\hline \multicolumn{3}{|c|}{ EPISODIOS NACIONALES. TERCERA SERIE } \\
\hline Zumalacárregui & 1898 & 71.013 \\
\hline Mendizábal & 1898 & 83.240 \\
\hline De Oñate a La Granja & 1898 & 80.346 \\
\hline Luchana & 1899 & 93.695 \\
\hline La campaña del Maestrazgo & 1899 & 73.299 \\
\hline La estafeta romántica & 1899 & 67.799 \\
\hline Vergara & 1899 & 77.776 \\
\hline Montes de Oca & 1900 & 61.666 \\
\hline Los Ayacuchos & 1900 & 75.899 \\
\hline Bodas reales & 1900 & 74.812 \\
\hline \multicolumn{3}{|c|}{ EPISODIOS NACIONALES. CUARTA SERIE } \\
\hline Las tormentas del 48 & 1902 & 73.726 \\
\hline Narváez & 1902 & 82.797 \\
\hline Los duendes de la camarilla & 1903 & 72.494 \\
\hline La Revolución de Julio & $1903-04$ & 77.393 \\
\hline O’Donnell & 1904 & 78.986 \\
\hline Aita Tettauen & 1904-05 & 78.507 \\
\hline Carlos VI en la Rápita & 1905 & 72.412 \\
\hline
\end{tabular}




\begin{tabular}{|c|c|c|}
\hline La vuelta al mundo en la Numancia & 1906 & 70.956 \\
\hline Prim & 1906 & 79.099 \\
\hline La de los tristes destinos & 1907 & 87.885 \\
\hline \multicolumn{3}{|c|}{ EPISODIOS NACIONALES. QUINTA SERIE } \\
\hline España sin Rey & 1907-08 & 78.784 \\
\hline España trágica & 1909 & 76.574 \\
\hline Amadeo I & 1910 & 73.434 \\
\hline La Primera República & 1911 & 68.228 \\
\hline De Cartago a Sagunto & 1911 & 68.285 \\
\hline Cánovas & 1912 & 68.306 \\
\hline \multicolumn{3}{|c|}{ NOVELAS SERIE PRIMERA ÉPOCA } \\
\hline La Fontana de Oro & 1870 & 125.722 \\
\hline La sombra; Celín; Tropiquillos; Theros & 1870 & 48.343 \\
\hline El audaz: historia de un radical de antaño & 1871 & 119.174 \\
\hline Doña Perfecta: novela original & 1876 & 65.486 \\
\hline Gloria & $1876-77$ & 127.697 \\
\hline Marianela & 1878 & 51.234 \\
\hline La familia de León Roch & 1878 & 145.720 \\
\hline \multicolumn{3}{|c|}{ NOVELAS SERIE CONTEMPORÁNEA } \\
\hline La desheredada & 1881 & 141.130 \\
\hline El amigo Manso & 1882 & 90.703 \\
\hline El doctor Centeno & 1883 & 113.949 \\
\hline Tormento & 1884 & 83.370 \\
\hline La de Bringas & 1884 & 71.862 \\
\hline Lo prohibido & $1884-85$ & 172.610 \\
\hline Fortunata y Jacinta: (dos historias de casadas) & $1886-87$ & 379.607 \\
\hline Miau & 1888 & 97.628 \\
\hline La incógnita & 1889 & 75.503 \\
\hline Realidad: novela en cinco jornadas & 1889 & 76.091 \\
\hline $\begin{array}{l}\text { Torquemada en la hoguera; El artículo de fondo; La mula y el } \\
\text { buey; La pluma en el viento; La conjuración de las palabras; } \\
\text { Un tribunal literario; La princesa y el granuja; Junio }\end{array}$ & 1889 & 62.658 \\
\hline Torquemada en la Cruz & 1893 & 58.302 \\
\hline
\end{tabular}




\begin{tabular}{lcc}
\hline Torquemada en el Purgatorio & 1894 & 67.625 \\
\hline Torquemada y San Pedro & 1895 & 61.367 \\
\hline Ángel Guerra & $1890-91$ & 249.534 \\
\hline Tristana & 1892 & 52.808 \\
\hline La loca de la casa: comedia en cuatro actos & 1892 & 36.527 \\
\hline Nazarín & 1895 & 65.019 \\
\hline Halma & 1895 & 76.162 \\
\hline Misericordia & 1897 & 84.131 \\
\hline El abuelo: (novela en cinco jornadas) & 1897 & 68.161 \\
\hline Total palabras & & 6.443 .709 \\
\hline
\end{tabular}

\section{Análisis}

El análisis se articula en torno a la proyección del discurso de los personajes por parte de los narradores de las distintas novelas de Galdós. De forma más concreta, el estudio se centra en la forma verbal dijo. Además de una cuestión de concreción metodológica, como se ha comentado en el apartado 1, la razón para que esto sea así estriba en la presencia destacada de esta forma en la obra del autor canario con respecto al resto de la novela realista española. Esta diferencia se muestra en la ilustración 1 (en la página siguiente), en la que aparecen dos capturas de pantalla, una con una lista de palabras extraída del corpus de estudio y otra con una lista de palabras extraída de un corpus de referencia formado por novelas realistas españolas 5 .

Como se puede advertir en la imagen, Galdós utiliza la forma dijo más del doble $(0,26 \%)$ que la novela realista en general (0,10\%). El uso destacado por parte de Galdós sugiere que esta forma verbal puede desempeñar una función estilística significativa en su obra. La mayoría de los ejemplos de dijo localizados en el corpus galdosiano se corresponden con la estrategia de representación del discurso de estilo directo, la más común en el género novelesco

5 El corpus de referencia con el que se ha comparado el corpus de estudio está formado por ochenta novelas de ocho autores decimonónicos españoles, pertenecientes también al Realismo: Pedro Antonio de Alarcón, Vicente Blasco Ibáñez, Leopoldo Alas “Clarín”, Luis Coloma, Armando Palacio Valdés, Emilia Pardo Bazán, José María de Pereda y Juan Valera. Las ochenta novelas tienen una extensión similar al corpus de Galdós, con una extensión de 6.188.478 palabras. Para una información más detallada del corpus, véase Nieto Caballero y Ruano San Segundo (en evaluación), en donde se utiliza como referencia en el análisis de algunos rasgos idiosincrásicos del estilo de Galdós en el marco de la novela realista española. 


\section{ILUSTRACIÓN 1}

Frecuencia de dijo en la obra de Galdós y en la novela realista

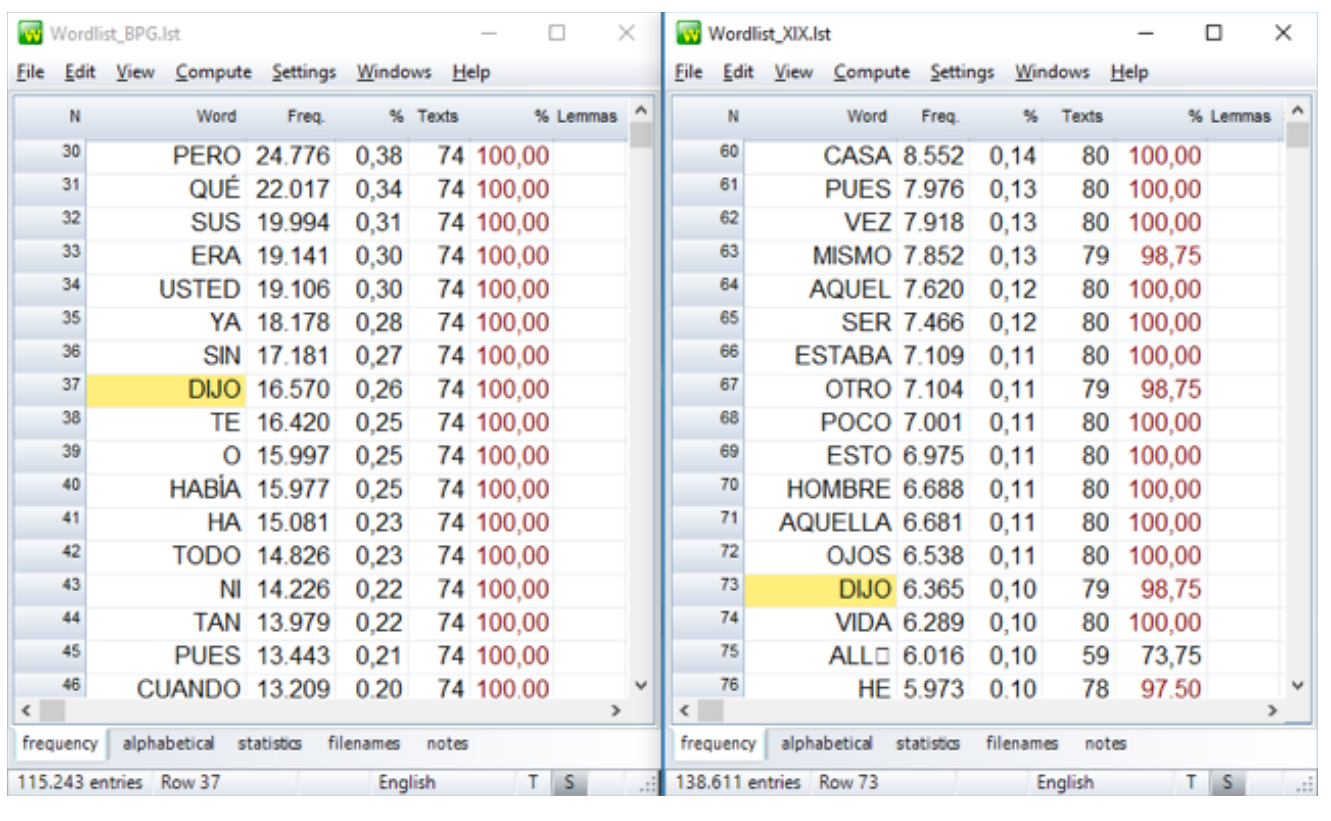

debido a su carácter mimético (Semino y Short, 2004: 97). Sin embargo, la distribución de los 16.570 ejemplos localizados no es ni mucho menos equitativa a lo largo del corpus. Como se muestra a continuación, la distribución irregular del verbo de habla por excelencia permite identificar - y calibrar - un cambio de paradigma en la representación del discurso de los personajes galdosianos a medida que se suceden las novelas, lo que revela a su vez aspectos estilísticos susceptibles de análisis que entroncan con la evolución literaria mencionada en el apartado 2. A continuación se muestra la distribución de la forma dijo en el corpus, para posteriormente analizar sus funciones y valorar la comentada evolución en términos estilísticos que se advierte en la producción narrativa del autor canario.

\subsection{Fase inicial y época de madurez}

El procesamiento del corpus con WordSmith Tools permite medir la representatividad de dijo en cada una de las setenta y cuatro novelas, lo que revela la mencionada irregularidad en la distribución de la forma verbal a lo largo de la obra de Galdós. Esta se advierte con claridad en la ilustración 2, en la que se muestra la frecuencia porcentual de dijo en cada una de las setenta y cuatro novelas, que aparecen dispuestas en orden cronológico según su fecha de publicación. Como se puede observar, en sus primeros años, el autor canario hace un uso evidentemente mayor del verbo de habla, que se atenúa sustancialmente a medida que suceden las novelas. 


\section{ILUSTRACIÓN 2}

Frecuencia (\%) de dijo en el corpus

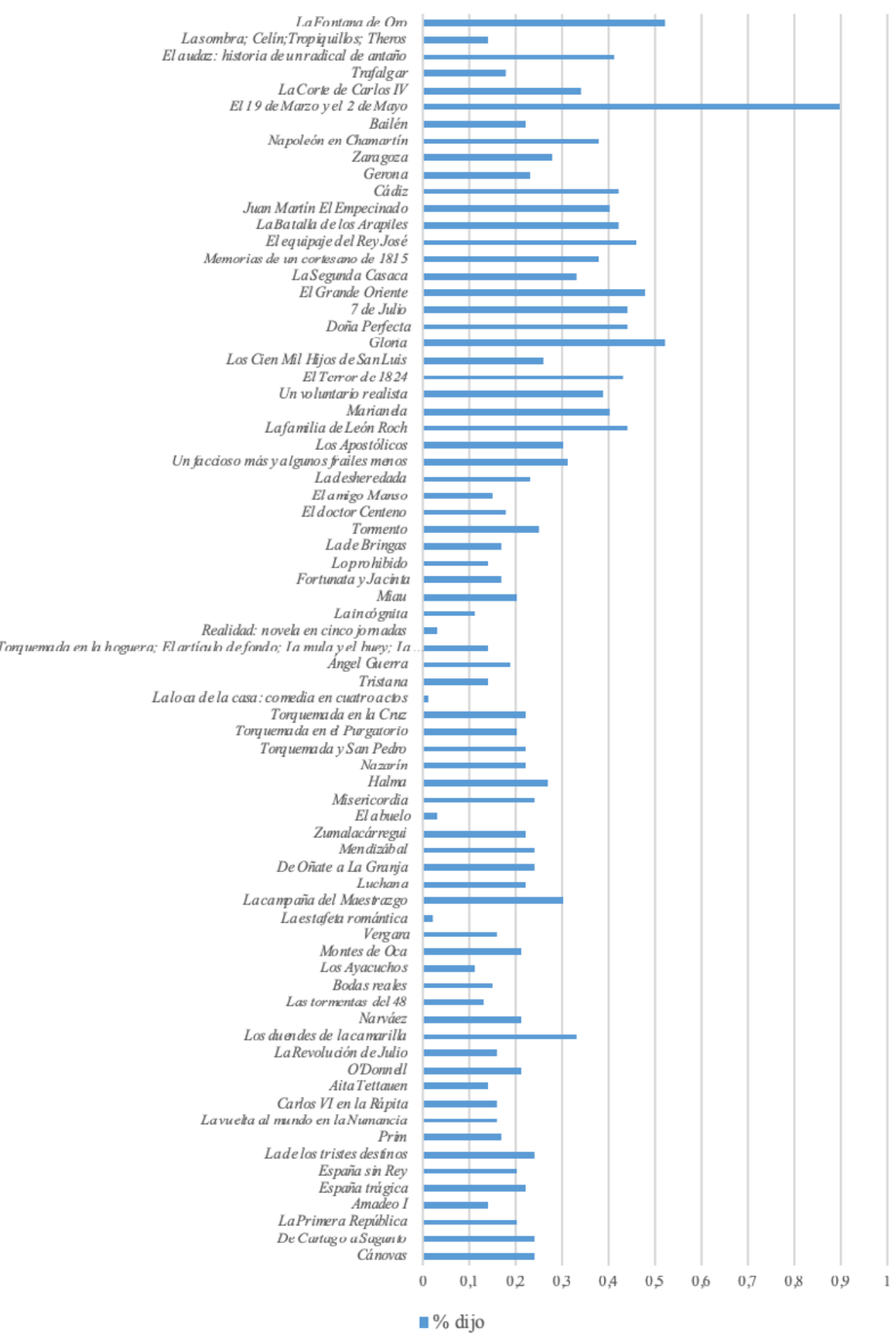


La información proporcionada en la ilustración resulta muy elocuente, pues no solo apunta la tendencia a la baja por parte de Galdós en el uso de dijo, sino que además revela una suerte de cambio de paradigma en el uso que el autor hace de la representación del discurso verbal en un momento concreto de su trayectoria. Como se puede advertir, en el primer tercio de su producción (aproximadamente) existe un uso destacado de dijo. Hacia La desheredada, sin embargo, la frecuencia en el uso que Galdós hace del verbo de habla decrece, para mantenerse estable - con destacadas excepciones - hasta el final de su producción. Resulta interesante que La desheredada sea la primera novela de la serie contemporánea (véase la tabla 1). Esta novela fue publicada en 1881. A partir de entonces, Galdós publicaría no solo el resto de novelas de la serie contemporánea, sino también todos los títulos que conforman la tercera, la cuarta y la quinta serie de los Episodios Nacionales. Previas a 1881 son, por otro lado, las novelas de la primera época y la primera y la segunda serie de los Episodios Nacionales. Esta suerte de punto de inflexión que marca el año 1881 con La desheredada se refuerza si calculamos la frecuencia de dijo en las novelas publicadas antes y después de 1881. Esta información aparece resumida en la tabla 2, en la que se muestra, en tantos por ciento, la frecuencia media del uso que Galdós hace de la forma verbal en ambos estadios de su producción.

\section{TABLA 2}

Frecuencia de dijo en las novelas de Galdós publicadas antes y después de 1888

\begin{tabular}{ccc}
$\begin{array}{c}\text { PERIODO DE } \\
\text { TIEMPO }\end{array}$ & SERIES & $\begin{array}{c}\text { FRECUENCIA } \\
\% \text { DIJO }\end{array}$ \\
$1878-1881$ & $\begin{array}{c}\text { Novelas serie primera época } \\
\text { Episodios Nacionales. Primera serie } \\
\text { Episodios Nacionales. Segunda serie }\end{array}$ & 0,37 \\
\hline $1881-1905$ & Novelas de serie contemporánea \\
& Episodios Nacionales. Tercera serie \\
& Episodios Nacionales. Cuarta serie \\
& Episodios Nacionales. Quinta serie & 0,19 \\
\hline
\end{tabular}

En virtud de la información proporcionada en la tabla podemos distinguir una fase inicial en la producción de Galdós bien diferenciada del resto de su trayectoria, al menos en lo que a representación del discurso verbal se refiere. En esta fase inicial el autor utiliza la forma dijo en una media de aproximadamente el doble (0,37\%) que en el resto de sus obras (0,19\%). El hecho de que la distinción entre ambos estadios de la producción del autor canario la marque La desheredada no parece ser fruto de la casualidad. Como se comentaba en el apartado 2, esta novela se caracteriza por un cambio de paradigma en la técnica narrativa de Galdós, siendo considerada incluso por muchos la primera novela naturalista. Como se ha comentado a raíz del ejemplo (1), el autor recurre a técnicas narrativas como el monó- 
logo interior, el estilo indirecto libre, el multiperspectivismo, la segunda persona en la voz del narrador o la inclusión de capítulos enteros dialogados. Este estilo, articulado en gran medida en torno al plano psicológico de los personajes, supone un cambio con respecto a la etapa inicial y se traduce en una pérdida de protagonismo de la voz del narrador, empleado de forma destacada por Galdós en sus comienzos para dar voz a sus personajes, como se mostraba en el ejemplo (2). Gracias a una metodología de corpus, este cambio de paradigma puede calibrarse con un alto grado de precisión, como se acaba de observar. Esta metodología, además, permite analizar sistemáticamente la figura del narrador en momentos dialógicos, lo que revela rasgos estilísticos típicos del Galdós de la primera época, que van aparejados al uso prominente de la figura del narrador para dar voz a sus personajes, como se muestra a continuación. En primer lugar, examinaremos el uso de bloques textuales relacionados con la construcción de los universos ficticios, para luego abordar la caracterización de los personajes, uno de los aspectos por los que Galdós goza de mayor reconocimiento.

\subsection{Construcción de los universos ficticios}

En el género novelesco, la voz del narrador en la estrategia de estilo directo no solo se emplea para introducir el discurso de los personajes, sino que también puede desempeñar funciones relevantes desde un punto de vista estilístico. En el caso de los ejemplos localizados en el corpus a raíz del uso que Galdós hace de la forma verbal dijo, el narrador galdosiano, al introducir el discurso de los personajes que pueblan la historia, ofrece frecuentemente información que contribuye a construir el universo ficticio que el autor nos presenta. La preponderancia del estilo directo en la primera época se materializa, de hecho, en un rasgo idiosincrásico del autor en su fase temprana a la hora de armar ese universo ficticio. Tómese el caso de Gloria como ejemplo, publicada en 1876. Galdós recurre hasta en ochenta y tres ocasiones a la fórmula dijo Gloria para introducir las palabras de la protagonista en estilo directo a través de la figura del narrador, como se puede observar en la ilustración 3, en la que se muestra una captura de pantalla con veinte de esos ochenta y tres ejemplos.

En esos ochenta y tres ejemplos, encontramos varios en los que el narrador, además de introducir el discurso de Gloria, incorpora información relevante acerca de elementos sobre los que se construye la historia. Esta información es fundamentalmente de dos tipos. Por un lado, junto a la representación del habla del personaje, el narrador suele describir el lenguaje gestual de este, lo que contribuye a modelar al personaje en el plano textual. Así, en sus intervenciones, vemos a Gloria sentándose (ejemplo 3) y levantándose (ejemplo 4), cruzando los brazos (ejemplo 5) o poniéndolos en cruz (ejemplo 6), tomando la mano de su amigo (ejemplo 7) o acariciando a unos chicos (ejemplo 8). El hecho de ofrecer esta información al tiempo que se introduce el habla del personaje crea una sensación se simultaneidad entre habla y movi- 


\section{ILUSTRACIÓN 3}

Captura de pantalla con veinte ejemplos de la concordancia dijo Gloria

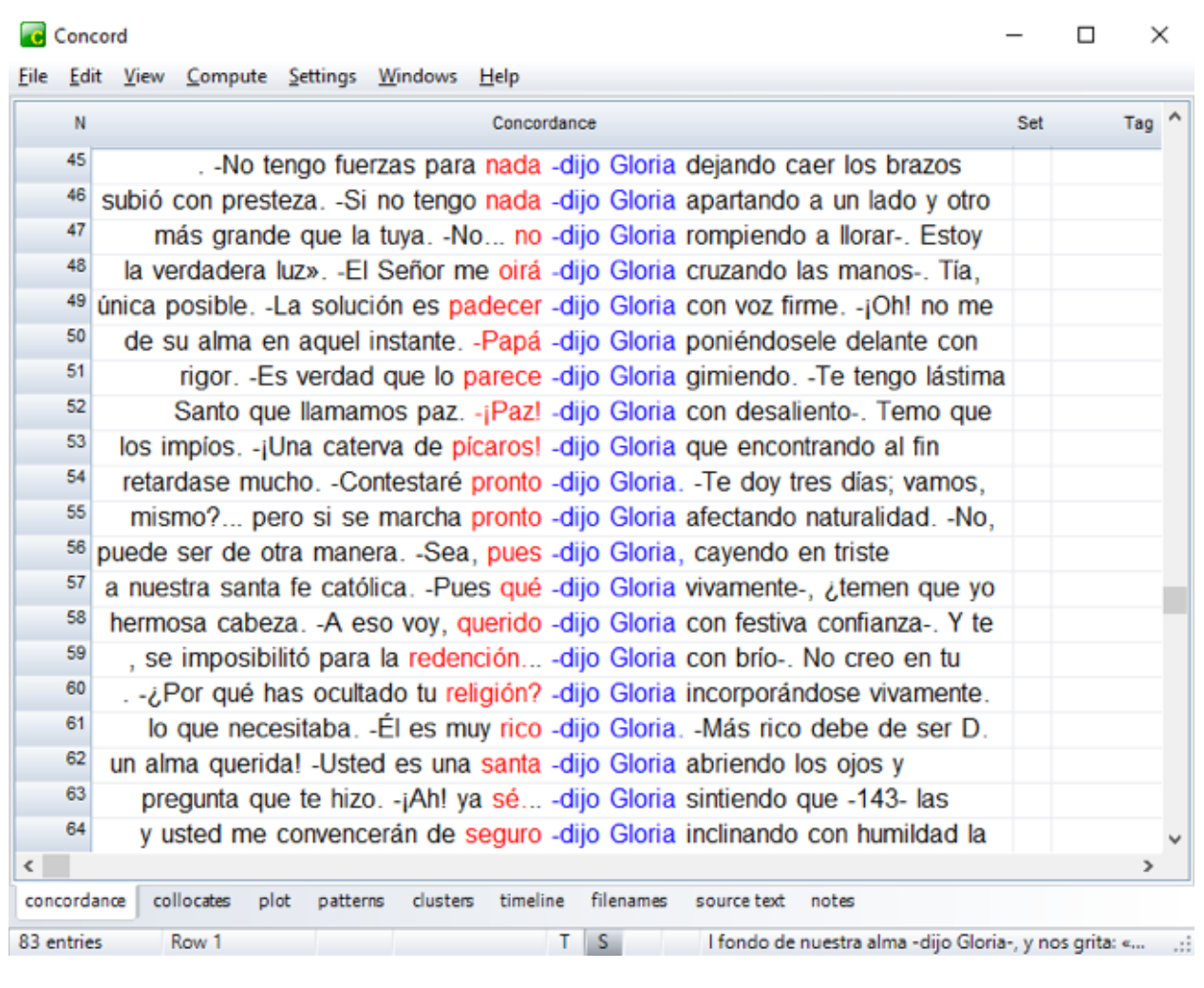

miento que potencia el carácter animado del personaje, lo que dota de realismo a la figura de Gloria en el marco del universo ficticio que Galdós nos plantea6.

(3) -Yo, yo sola —dijo Gloria sentándose también-, soy la culpable. Hace tiempo, desde que le conocí, dime a cavilar en estas cosas noche y día. No podía apartarlas de mi pensamiento y, según mi entender, discurría acertadamente sobre ellas. Me parecía que mis argumentos no tenían réplica, y me vanagloriaba de ellos pronunciándolos en mis diálogos oscuros conmigo misma (Gloria, capítulo 30).

(4) - ¿Por qué has ocultado tu religión? —dijo Gloria incorporándose vivamente (Gloria, capítulo 37).

(5) - El Señor me oirá — dijo Gloria cruzando las manos- Tía, querida tía, mi alma se llena repentinamente de fe; en mí ha entrado una luz prodigiosa; siento como una gran Iluvia... Soy otra... Suena dentro de mí una voz como el trueno... Me parece que Dios me dice: Sí, sí, sí (Gloria, capítulo 21).

6 Para un análisis detenido del papel del lenguaje gestual en el marco de la construcción de los personajes de ficción, y de cómo este se relaciona con la voz del narrador, véase Korte (1997). 
(6) —iCrucificarme! ¿No lo estoy ya? —dijo Gloria extendiendo los brazos en cruz (Gloria, capítulo 15).

(7) - Un favor te pido, segura de que me lo has de conceder — dijo Gloria, tomando la mano de su amigo (Gloria, capítulo 32).

(8) - Por Dios, José, me estás asustando — dijo Gloria acariciando a los chicos que se habían echado a llorar viendo llorar al padre-. Si es verdad lo que dices, eres un hombre de muy mala conducta (Gloria, capítulo 15).

Por otro lado, la información también tiene que ver frecuentemente con elementos de la escena. Tales son los casos de los ejemplos (9) y (10), en los que se introducen en la historia el taller de Caifás y una piedra que hay al lado de una casa, sobre la que se sienta Gloria. Este tipo de ejemplos encajan en el domino del espacio visual y el movimiento referido normalmente por la crítica, con el que "se prefigura el ritmo cinematográfico" que caracterizará sus novelas (Arroyo Díez, 2011: 366). En su etapa inicial, Galdós aborda muchas de las cuestiones relacionadas con el espacio visual precisamente en momentos dialógicos. En ocasiones, además, dado que la voz del narrador se utiliza en primera instancia para reproducir el discurso de un personaje, la información se le ofrece al lector a través de la mirada del propio personaje. Esto es lo que ocurre en los ejemplos (11) y (12), en donde descubrimos, a través de los ojos de Gloria, la distancia entre dos personajes o los lugares para tomar asiento en una habitación. Utilizar la mirada de un personaje para que, como lectores, configuremos la escena que se nos presenta resulta común en el género novelesco en general (cf. Korte, 1997: 57). En el caso de Galdós y sus primeras novelas, además, se advierte una relación directa entre este aspecto y la representación del discurso de los personajes por parte del narrador.

(9) —iQué espantoso temporal! —dijo Gloria entrando en el taller de Caifás (Gloria, capítulo 15).

(10) -Esas risotadas y esas cabriolas — dijo Gloria sentándose en una piedra que junto a la casa había-, no sientan bien en la persona de un desgraciado que acaba de sufrir tan terribles golpes (Gloria, capítulo 25).

(11) —iOh! iqué bien estoy aquí! —dijo Gloria mirando a los ojos de su amigo a distancia de pocos dedos-. ¡Mi hijo! itú!... lo que más amo en el mundo (Gloria, capítulo 32).

(12) —No, esperaré a que pase el agua. De casa vendrán por mí —dijo Gloria, buscando con los ojos un sitio donde sentarse (Gloria, capítulo 15).

En suma, la voz protagonista del narrador en la fase inicial de la trayectoria de Galdós se traduce en una marcada relación entre la proyección del habla de los personajes y aspectos relacionados con la construcción del universo ficticio. Esta relación, materializada en bloques textuales que acompañan a la fórmula "dijo + nombre del personaje”, se convierte en un rasgo idiosincrásico del estilo de Galdós en su primera época, para atenuarse gradualmente 
a medida que la representación del discurso verbal a través de la forma dijo va perdiendo presencia en la producción del autor, en favor de otras estrategias de representación del discurso, como el monólogo interior o el estilo indirecto libre. Naturalmente, este descenso no equivale necesariamente a una disminución en las intervenciones de los personajes en la historia - El abuelo, sin ir más lejos, es una novela narrada al modo de una obra de teatro, en donde el discurso de los personajes ocupa la gran mayoría del texto-, sino a un cambio de paradigma en las opciones ejercidas para representar el discurso de estos. Este cambio se ve reflejado en el descenso paulatino de técnicas narrativas como el estilo directo, que acarrean implicaciones estilísticas claras, como acaba de comprobarse. Gracias a una metodología de corpus, estas implicaciones pueden analizarse sistemáticamente a lo largo de la trayectoria del autor.

\subsection{Caracterización}

Por otro lado, la voz protagonista del narrador también desempeña funciones textuales locales (local textual functions) (Mahlberg, 2007: 195) en novelas de la fase inicial. Un ejemplo claro es el de la caracterización de los personajes. Mientras que en su época de madurez, debido al auge de técnicas como el estilo indirecto libre o el monólogo interior, el plano mental de los personajes se fundamenta sobre todo en la proyección de sus pensamientos, en sus primeros años Galdós se beneficia del patrón estructural del estilo directo para ofrecer al lector información relacionada con la dimensión psicológica de los personajes a través de la voz del narrador. Como afirma Caldas-Coulthard, en el caso de verbos neutrales en textos de ficción, como el ejemplo de decir que aquí se utiliza como punto de partida en el análisis, los narradores suelen "gloss utterances with the reporting verb 'say' plus either an adverb, an adjective, or a prepositional clause which will mark either manner or attitude” (Caldas-Coulthard, 1988: 167). Este tipo de información puede, naturalmente, ser importante en términos de caracterización a través de la evaluación (Hunston y Thompson, 2000) del acto de habla que se representa. La preponderancia de la forma verbal dijo para introducir discurso en los primeros años de su producción se materializa nuevamente en un rasgo idiosincrásico del estilo de Galdós en su primera época. El caso de Clara en La Fontana de Oro es un buen ejemplo. Gracias a un enfoque de corpus, las valoraciones del narrador sobre los actos de habla de este personaje pueden analizarse sistemáticamente. Una concordancia de dijo Clara en el corpus de estudio, por ejemplo, identifica todos los ejemplos en una sola lista, lo que permite una lectura vertical de estos (Tognini-Bonelli, 2001: 18). Esta lectura vertical hace posible, a su vez, identificar patrones recurrentes que contribuyen a perfilar el plano mental del personaje. En la ilustración 4 se muestran todos los ejemplos de dijo que se asocian con este personaje en estilo directo. 


\section{ILUSTRACIÓN 4}

Captura de pantalla con resultados de la concordancia dijo Clara

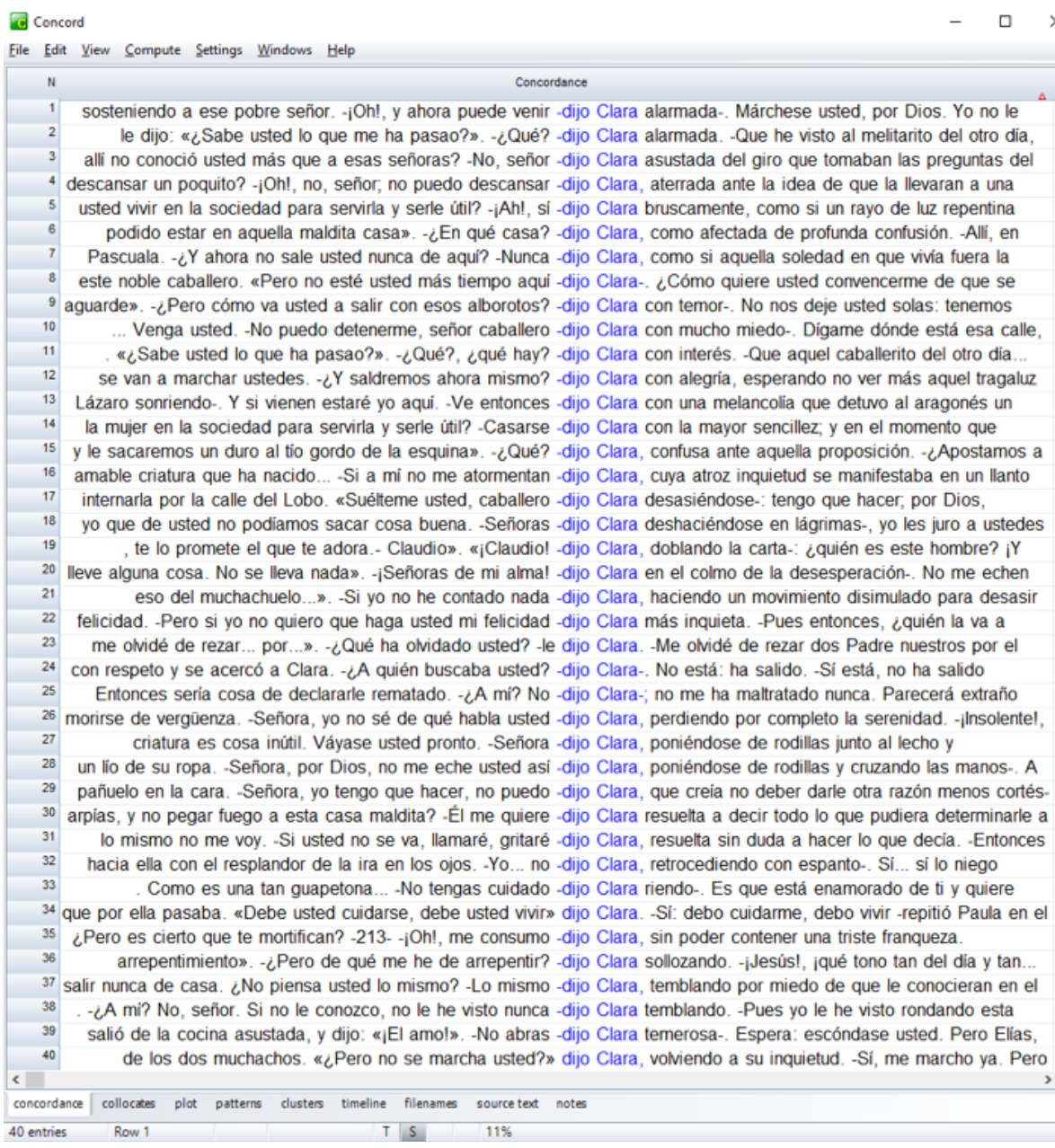

Como se puede advertir en la ilustración, el modo en que Clara dice las cosas tiene un denominador común: su temor, uno de sus rasgos idiosincrásicos que mejor la definen. Sirvan las cuatro primeras concordancias como ejemplo. En dos ocasiones pronuncia sus palabras alarmada, en otra ocasión asustada y en otra aterrada, como se puede observar en los ejemplos (13) a (16):

(13) —iOh!, y ahora puede venir — dijo Clara alarmada—. Márchese usted, por Dios. Yo no le conozco, ni me importa todo eso que me ha dicho. Si él llega... (La Fontana de Oro, capítulo 13).

(14) - ¿Qué? - dijo Clara alarmada (La Fontana de Oro, capítulo 8).

(15) - No, señor - dijo Clara asustada del giro que tomaban las preguntas del clérigo (La Fontana de Oro, capítulo 38) 
(16) — ¡Oh!, no, señor; no puedo descansar — dijo Clara, aterrada ante la idea de que la llevaran a una sacristía (La Fontana de Oro, capítulo 38).

Además, sus actos de habla también destacan por estar articulados con mucho miedo (concordancia 10), con temor (concordancia 9), con melancolía (concordancia 13), deshaciéndose en lágrimas (concordancia 18), perdiendo la serenidad (concordancia 26), retrocediendo con espanto (concordancia 32), sin poder contener una triste franqueza (concordancia 35), soIlozando (concordancia 36), temblando (concordancia 38) o temerosa (concordancia 39). Es cierto que, por sí solas, cada una de las proposiciones proyectoras que sirven para introducir su discurso parece simplemente contextualizar el acto de habla, describiendo la situación en la que se inserta a través de una explicación de los detalles que la definen. En conjunto, sin embargo, la información que sistemáticamente recibimos como lectores contribuye a proyectar el carácter de Clara. De forma más precisa, la evaluación del personaje a través de la voz del narrador contribuye a la creación de un "cumulative effect” (Montoro, 2012: 99) que sirve para completar (o mejor dicho, reforzar) la caracterización de Clara en el transcurso de la historia, pues, como se puede observar en la ilustración 5, los ejemplos localizados aparecen dispuestos a lo largo de la novela, y no en un momento concreto de esta.

\section{ILUSTRACIÓN 5}

Distribución de dijo Clara en La fontana de oro

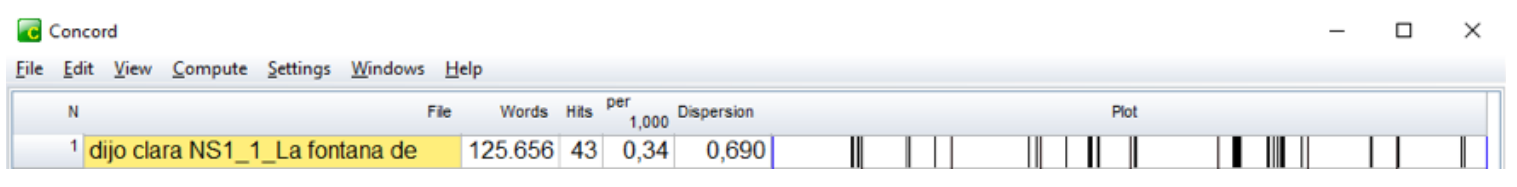

Esta disposición, así como su valor caracterizador, entronca directamente con enfoques propios de la estilística cognitiva (West, 2016) y con teorías sobre la percepción de los personajes de ficción, como el "mind-modelling" (Stockwell, 2009)7. Debido sobre todo a "the attention readers devote to psychological states of the main characters" (el énfasis es mío) (Hakemulder, 2016: 196), la repetición en el transcurso de la historia de la información sobre el carácter de Clara proporcionada en sus intervenciones puede ayudar a definir la imagen que Galdós proyecta de ella durante la historia y que, como lectores, nos formamos en el acto de lectura de la novela. Naturalmente, se trata de un análisis complejo, que requiere una profundidad que rebasaría los límites de este estudio. En cualquier caso, la sistematicidad con la que este tipo

7 En términos generales (esto es, más allá del ámbito literario que aquí nos ocupa), el mind-modelling puede definirse como el "process by which we perceive and understand minds other than our own" (Harrison, 2017: 30). 
de información caracterizadora sobre los personajes se encuentra en las proposiciones que introducen el discurso de estos en las novelas de la fase inicial de Galdós revela una opción ejercida por el autor canario que acarrea implicaciones estilísticas, como acaba de verse a través del ejemplo de Clara. Debido al cambio de paradigma en la representación del discurso de los personajes que se viene comentando, esta técnica de caracterización se atenúa con el paso del tiempo e ilustra nuevamente la evolución en el estilo de Galdós apuntada por la crítica y que aquí se analiza.

En suma, tanto la caracterización de sus figuras como los bloques textuales de los que se sirve para construir el universo ficticio de sus historias analizado en el apartado 4.2 sirven para trazar una evolución en el estilo de Galdós. Estos aspectos, muy presentes en la fase inicial de su trayectoria, pierden fuerza en su época de madurez, donde el uso de nuevas técnicas narrativas para indagar en la interioridad de los personajes se impone a medida que se suceden las novelas.

Cabe destacar, a modo de cierre, que la identificación de estos aspectos en la fase inicial del Galdós, así como su análisis sistemático, ha sido posible gracias a un enfoque de corpus, con el que se ha podido medir la distribución del uso que el autor canario hace de dijo a lo largo de sus setenta y cuatro novelas. En este sentido, el presente artículo ha pretendido ilustrar el potencial de los estudios de corpus en el análisis de textos literarios en lengua española, un área todavía escasa de investigaciones que adopten este tipo de enfoque. Como se ha podido observar, una metodología de corpus como la que aquí se ha empleado no solo es útil para "confirm findings from many years of traditional study" (Stubbs, 2005: 6), sino también para calibrarlos con mayor precisión y analizarlos de forma metódica a través de aspectos que tradicionalmente han pasado desapercibidos para la crítica.

\section{Conclusión}

En este artículo se ha presentado una propuesta para el análisis de la evolución literaria de un autor utilizando herramientas propias de la lingüística de corpus. En concreto, se ha analizado la figura de Benito Pérez Galdós utilizando un enfoque de estilística de corpus. El análisis ha girado en torno a la proyección del discurso verbal de los personajes del universo galdosiano por parte de los narradores de las distintas novelas, tomando como punto de partida la forma verbal dijo, verbo de habla por excelencia en la representación del discurso en el género novelesco. El objetivo del estudio ha sido doble. En primer lugar, se ha intentado calibrar la evolución estilística del autor a través de la representación del discurso de sus personajes. Los resultados obtenidos han revelado un marcado uso de la forma dijo en las primeras novelas, que ha permitido establecer una división clara entre la fase inicial y la de madurez. El uso destacado de dijo en la primera etapa encaja con el protagonismo de la voz del narrador 
al que suele hacer referencia la crítica especializada en los inicios de Galdós. Este uso decrece con el paso del tiempo y, a partir de La desheredada, se advierte un uso sustancialmente menor que encaja con la utilización de nuevas técnicas narrativas (el estilo indirecto libre, el monólogo interior, etc.) empleadas por Galdós para indagar en el plano psicológico de sus personajes - no en vano La desheredada es para muchos la primera novela naturalista- Sin embargo, el análisis del uso que Galdós hace de dijo no solo sirve para ilustrar este cambio de paradigma, sino que también revela opciones ejercidas sistemáticamente por el autor en la primera época, que definen claramente el estilo de su fase inicial. Así, el uso destacado de dijo se materializa en un sincretismo en la reproducción del discurso y la construcción del universo ficticio que nos presenta a través de la voz del narrador. De la misma manera, es a través de la figura protagonista del narrador como Galdós ofrece al lector información relacionada con la dimensión psicológica de los personajes, un aspecto que más adelante se aborda mediante el uso de otras técnicas. El análisis de estos aspectos revela una evolución en el estilo del autor canario, que ha sido posible definir y calibrar gracias a herramientas propias de la lingüística de corpus. Y es ahí donde se cifra el segundo objetivo de este análisis: demostrar el potencial de la estilística de corpus como disciplina de estudio en el análisis de textos literarios en español. Desafortunadamente, y aunque se trata de un enfoque habitual en el análisis de textos literarios en otras lenguas, este tipo de metodologías son escasas por no decir inexistentes - en el análisis de textos literarios en lengua española. Esta falta de estudios parece tener su origen, al menos parcialmente, en las reticencias de una crítica literaria contraria al componente cuantitativo de las metodologías de los estudios de corpus. Sin embargo, este no es un enfoque que pretenda reemplazar a otros más tradicionales. Por el contrario, las metodologías de corpus deben ser consideradas como enfoques complementarios, de los que el análisis de textos literarios se puede beneficiar en gran medida, como esperamos haber demostrado en este artículo.

\section{Bibliografía citada}

Andreu, Alicia G., 1989: Modelos dialógicos en la narrativa de Benito Pérez Galdós, Amsterdam: John Benjamins Publishing.

Arrovo Diez, María Cristina, 2011: Aspectos espaciales y visuales en las primeras novelas contemporáneas Benito Pérez Galdós y su repercusión en la novela española actual. Tesis de maestría, Universidad de Valladolid.

Benitez, Rubén, 1992: La literatura española en las obras de Galdós, Murcia: Universidad de Murcia.

Biblioteca Virtual Miguel de Cervantes (s. f.): "Repositorio digital” [http://www.cervantesvirtual. com/, fecha de consulta: 1 de septiembre de 2017]. 
Caldas-Coulmthard, Carmen Rosa, 1988: Reporting Interaction in Narrative: a Study of speech presentation in written discourse. Tesis de maestría, University of Birmingham.

Gullón, Ricardo, 1960: Galdós. Novelista moderno, Madrid: Taurus.

Gutiérrez Carbajo, Francisco, 2013: Movimientos y épocas literarias, Madrid: Universidad Nacional de Educación a Distancia.

HAKEMUlder, Frank, 2016: "Empirical Stylistics" en Violeta SotiRova (ed.): The Bloomsbury Companion to Stylistics, London \& New York: Bloomsbury, 198-207.

HaRrIson, Chloe, 2017: Cognitive Grammar in Contemporary Fiction, Amsterdam: John Benjamins.

Hughes, James M., Nicholas Fotı, David C. Krakauer y Daniel N. Rockmore, 2012: "Quantitative patterns of stylistic influence in the evolution of literature", Proceedings of the National Academy of Sciences 109 (20), 7682-7686.

Hunston, Susan, y Geoff Thompson, 2000: Evaluation in Text: Authorial Stance and the Construction of Discourse, Oxford: Oxford University Press.

KORTE, Barbara, 1997: Body Language in Literature, Toronto: University of Toronto Press.

LAmBERT, Mark, 1981: Dickens and the Suspended Quotation, New Haven: Yale University Press.

LanCASHIRE, Ian, 1993: Computer-Based Chaucer Studies, Toronto: Centre for Computing in the Humanities.

Mahlberg, Michaela, 2007: "Clusters, key clusters and local textual functions in Dickens", Corpora $2(1), 1-31$.

MahlBerg, Michaela, 2016: "Corpus stylistics" en Violeta Sotirova (ed.): The Bloomsbury Companion to Stylistics, London \& New York: Bloomsbury, 139-156.

Mahlberg, Michaela, y Catherine SMith, 2012: "Dickens, the suspended quotation and the corpus", Language and Literature 21 (1), 51-65.

Mahlberg, Michaela, Catherine Smith y Simon Preston, 2013: "Phrases in literary contexts: Patterns and distributions of suspensions in Dickens's novels", International Journal of Corpus Linguistics 18 (1), 35-56.

Martínez-GamboA, Ricardo, 2015: "Patrones cuantitativos en novelas chilenas de los siglos XIX a XXI”, Onomázein 32, 239-253.

Martínez-Gamboa, Ricardo, 2016: "Big data en humanidades digitales: de la escritura digital a la 'lectura distante”, Revista Chilena de Literatura 94, 39-58. 
Montoro, Rocío, 2012: Chick Lit: The Stylistics of Cappuccino Fiction, London: Continuum.

Nieto Caballero, Guadalupe, y Pablo Ruano San Segundo, en evaluación: "Metodologías de corpus en el análisis de textos literarios en lengua española: el ejemplo de Pérez Galdós", RESLA.

Pedraza Jiménez, Felipe B., y Milagros Rodriguez Cáceres, 1983: Manual de literatura española. Tomo VII: Época del Realismo, Madrid: Cénlit Ediciones S. L.

ScotT, Mike, 2016: WordSmith Tools version 7, Stroud: Lexical Analysis Software.

Semino, Elena y Mick Short, 2004: Corpus Stylistics: Speech, Writing and Thought Presentation in a Corpus of English Narratives, London: Routledge.

Sotelo, María Luisa, 2013: Realismo y Naturalismo en España. La novela, Barcelona: Universitat de Barcelona.

Stockwell, Peter, 2009: Texture: A Cognitive Aesthetics of Reading, Edinburgh: University Press.

StuBbs, Michael, 2005: "Conrad in the computer: Examples of quantitative stylistics methods", Language and Literature 14 (1), 5-24.

Tognini-Boneluı, Elena, 2001: Corpus Linguistics at Work, Amsterdam: John Benjamins.

VARGas Llosa, Mario, 1975: La orgía perpetua. Flaubert y Madame Bovary, Barcelona: Seix Barral.

WeSt, David 2016: "Cognitive Stylistics" en Violeta SotIRova (ed.): The Bloomsbury Companion to Stylistics, London \& New York: Bloomsbury, 109-121. 